\title{
SIMPLIFICAÇÃO DO ÍNDICE CPO DOS 18 AOS 25 ANOS
}

Luiz O. C. Guimarães*

Ana M. R. Guimarães**

GUIMARÃES, L. O. C. \& GUIMARÃES, A. M. R. Simplificação do índice CPO dos 18 aos 25 anos. Rev. Saúde públ., S.Paulo, 24: 407-11, 1990.

RESUMO: O índice CPO pode ser estimado pelo dobro do valor obtido pelo exame de dois hemiarcos (superior direito e inferior esquerdo), e pelo quádruplo do valor observado em seis dentes (primeiro molar e incisivo central superiores direitos, primeiro pré-molar superior esquerdo, segundo molar e incisivo lateral inferiores esquerdos e segundo pré-molar inferior direito). As estimativas foram feitas por métodos da estatística descritiva, em termos de média, composição percentual e coeficiente de prevalência de cárie, tendo-se levantado dados em 100 indivíduos de 18 a 25 anos.

DESCRITORES: Indice CPO. Cárie dentária, epidemiologia.

\section{INTRODUÇÃO}

O índice CPO descrito por Klein e Palmer ${ }^{6}$ (1937) é o índice mais utilizado em levantamentos epidemiológicos para determinar a prevalência e a incidência da cárie dentária. É empregado também no planejamento e avaliação de programas de assistência odontológica em saúde pública, como ainda em pesquisas sobre métodos preventivos. Apresenta características favoráveis, sendo objetivo e simples, que determinaram sua utilização universal nesses cinqüenta anos.

Vários autores sugeriram modificações ou simplificaçoes do índice, entre os quais podemos citar East e Pohlen 2(1941), que propuseram expressar o índice em percentagem.

Sendo a cárie dentária um evento predominantemente bilateral, a prevalência da cárie e as necessidades de tratamento odontológico podem ser obtidas pelo exame de metade do arco superior e da metade oposta do arco inferior, o que foi observado por vários autores como Knutson e Armstrong ${ }^{9}$, Knutson e Klein ${ }^{7}$, Pancova e Mrklas ${ }^{12}$, Scott ${ }^{14}$ e Welander ${ }^{17}$. Esta simplificação foi acolhida pela Organização Mundial de Saúde ${ }^{18}$, em 1971, reafirmada em $1977^{19}$ e $1987^{20}$, sendo sugerido o rodízio no exame de hemiarcos opostos, superior e inferior; o rodízio pode acarretar dificuldades na execução do levantamento quando se utilizam vários examinadores.

Outra simplificação do índice $\mathrm{CPO}$, proposta por Knutson ${ }^{8}$, é baseada na relação entre o índice e a percentagem de indivíduos com pelo menos um dente permanente atacado pela cárie, determina- da em pessoas de 6 a 15 anos, sendo a regressão expressa pela equação: $97-y=97(0,524)^{x}$ onde y é a percentagem de indivíduos e $x$ o índice $C P O$. A estimativa pode ser útil para estimar o índice em áreas de baixa prevalência de cárie, até o limite de $90 \%$, que corresponde ao valor quatro para o indice $\mathrm{CPO}$.

Posteriormente Lilienthal e Andrews ${ }^{10}$ obtiveram regressões ligeiramente diferentes, em dados obtidos em Formosa, Indonésia e Austrália, concluindo, no entanto, pela aplicabilidade satisfatória da relação determinada por Knutson, para fins de saúde pública. Chaves reconhece que o valor prático da relação de Knutson é a possibilidade de estimar o índice $\mathrm{CPO}$, usando menos tempo e esforço.

Heer ${ }^{5}$ propôs uma simplificação baseada no exame de um dente em cada dentição (primeiro molar permanente e segundo molar decíduo inferiores do mesmo lado) que representariam todos os dentes. Viegas ${ }^{15}$ desenvolveu uma simplificação do índice utilizando o primeiro molar inferior direito para estimar a prevalência da cárie dentária em crianças; outra simplificação estudada por esse mesmo autor inclui o incisivo central superior. Lopes ${ }^{11}$ estudou a distribuição da variável obtida pelo método de Viegas, verificando que não difere significantemente da distribuição normal, mesmo em amostras reduzidas, concluíndo pela validade da utilização de métodos estatísticos paramétricos.

Guimarães ${ }^{3}$ estudou a simplificação do índice CPO pelo exame de 2 hemiarcos; o índice CPO, neste caso, é estimado pelo dobro do valor obtido

\footnotetext{
* Departamento de Prática de Saúde Pública da Faculdade de Saúde Pública da Universidade de São Paulo - Av. Dr. Arnaldo, 715 - 01255 - São Paulo, SP - Brasil.

* Instituto de Ciências da Saúde da Universidade Paulista - Rua Dr. Bacelar, 1.212 - 04026 - São Paulo, SP - Brasil.
} 
nos hemiarcos superior esquerdo e inferior direito; outra simplificação também estudada pelo citado autor baseia-se no exame de seis dentes: primeiro molar e incisivo central superiores direitos, primeiro pré-molar superior esquerdo, segundo molar e incisivo lateral inferiores esquerdos e o segundo pré-molar inferior direito. Observou ainda que a composição percentual do índice CPO é similar a observada em dois hemiarcos, ou seis dentes; esses estudos foram realizados em escolares de 7 a 12 anos.

Rodrigues ${ }^{13}$ (1987) estudou a simplificação do índice ceo, pelo exame de quatro dentes decíduos: primeiro molar superior direito, incisivo central superior esquerdo, segundo molar inferior esquerdo e incisivo lateral inferior direito; na dentição mista acrescentou dois dentes permanentes: o primeiro molar superior esquerdo e o primeiro molar inferior direito; recomenda ainda que na ausência do dente selecionado seja examinado o dente distal mais próximo e na ausência também deste, o dente mesial mais próximo daquele inicialmente indicado. Utilizou dados de 4 a 10 anos, na dentição decídua e mista, obtendo valores elevados para o coeficiente de correlação, evidenciando uma associação estatística entres essas variáveis.

O índice CPO é a soma dos dentes permanentes cariados, perdidos e obturados podendo ser relacionado ao número de dentes permanentes irrompidos, determinando-se Coeficiente de Prevalência de Cárie (CPC), proposto por Guimarães e col.4, que apresenta distribuição normal e homocedasticidade, permitindo a utilização de métodos estatísticos paramétricos. Este coeficiente é expresso em percentagem, o que facilita sua compreensão.

A maioria dos trabalhos publicados na literatura sobre a redução no exame de dentes estão baseados em dados de prevalência de cárie obtidos em crianças de 6 a 12 ou até os 15 anos.

O objetivo do presente trabalho é verificar a aplicabilidade da simplificação de índice CPO, pelos dados de dois hemiarcos e de seis dentes, em indivíduos de 18 a 25 anos de idade.

\section{MATERIAL E MÉTODOS}

Foram realizados exames odontológicos em 100 indivíduos entre 18 e 25 anos de idade, dos quais 41 do sexo masculino e 59 do feminino, alunos da Universidade Paulista (UNIP), que apresentam nível sócio-econômico médio ou elevado, e conhecimentos sobre a cárie dentária.

Os exames foram realizados com espelho e sonda exploradora, usando-se os consultórios odontologicos da UNIP; e os exames clínicos foram realizados das 16:30 às 19:30, o que determinou o uso padronizado de refletores.
Dois cirurgiōes dentistas, docentes de odontologia preventiva daquela Universidade, com larga experiência em levantamentos epidemiológicos, realizaram os exames clínicos, tendo sido previamente calibrados; a calibração foi realizada pelo exame de 10 voluntários e discussão das diferenças, para fins de padronizar o método de exame e critérios. Numa segunda etapa, outros 15 voluntários foram examinados sucessivamente pelos dois examinadores; as discordâncias foram $3 \%$, valor adequado às finalidades deste estudo e inferior ao padrão proposto pelo "Dental Health Center" $(5 \%)$, conforme menciona Chaves ${ }^{1}$.

Os 32 espaços das arcadas dentárias foram examinados, anotando-se os dados em ficha clínica individual, inclusive terceiros molares; os espaços foram identificados pela notação binária recomendada pela Federação Dentária Internacional, na qual o primeiro dígito identifica o hemiarco e, o segundo, o dente.

Cada espaço recebeu um diagnóstico, conforme o dente se apresentasse cariado, extraído, com extração indicada, obturado, hígido ou não irrompido, critérios mutuamente exclusivos. Foram anotados como espaço vazio os dentes ausentes por outras causas como, por exemplo, as anodontias e extrações por indicação ortodôntica.

Os participantes se inscreveram como voluntários e, os exames clínicos foram realizados em maio de 1988.

\section{RESULTADOS E DISCUSSÃO}

$\mathrm{O}$ índice $\mathrm{CPO}$ e componentes e a composição percentual do índice são apresentados na Tabela 1.

$O$ índice apurado nos hemiarcos superior direito e inferior esquerdo são apresentados na Tabela 2 , incluídos os dados dos outros dois hemiarcos, calculado segundo o sexo.

\section{TABELA 1}

Indice CPO e componentes, dentes hígidos e irrompidos em universitários de 18 a 25 anos, São Paulo, 1988

\begin{tabular}{lrcc}
\hline Dentes & Índice CPO & $\begin{array}{c}\text { Comp (CPO) } \\
\mathrm{A}^{* \%}\end{array}$ & $\begin{array}{c}\text { Comp (IRR) } \\
\mathrm{B}^{* \%}\end{array}$ \\
\hline Cariados & 1,3 & 9,6 & 4,6 \\
Obturados & 12,1 & 89,6 & 42,1 \\
Extraídos & 0,1 & 0,8 & 0,4 \\
Ext. Indicada & 0,0 & 0,0 & 0,0 \\
Índice CPO & 13,5 & 100,0 & 47,1 \\
Hígidos & 15,3 &.-- & 52.9 \\
Irrompidos & 28,8 & -- & 100,0 \\
\hline
\end{tabular}

*A - Em função dos dentes atacados (índice CPO).

*B - Em função dos dentes irrompidos. 
TABELA 2

Indice CPO em universitários de 18 a 25 anos, segundo o sexo e hemiarcos, São Paulo, 1988

\begin{tabular}{llcccc}
\hline \multirow{5}{*}{ Sexo } & \multicolumn{3}{c}{ Indice C.P.O } & & \\
\cline { 2 - 5 } & $\begin{array}{l}\text { Todos } \infty \\
\text { dentes }\end{array}$ & $\begin{array}{l}\text { Hemiarcos } \\
\text { S.D. + I.E. }\end{array}$ & $\begin{array}{l}\text { Hemiarcos } \\
\text { S.E. + I.D. }\end{array}$ & $\begin{array}{l}\text { CPO } \\
\text { est. }\end{array}$ & $\begin{array}{l}\text { Dif CPO } \\
\text { Obs - est. }\end{array}$ \\
\hline Masculino & 13,3 & 6,6 & 6,7 & 13,2 & 0,1 \\
Feminino & 13,7 & 6,9 & 6,8 & 13,8 & $-0,1$ \\
Ambos & 13,5 & 6,8 & 6,7 & 13,6 & $-0,1$ \\
\hline
\end{tabular}

* Hemiarcos (SD + IE) $\times 2$

TABELA 3

Composiçăo percentual do indice de CPO e hermiarcos superior direito e inferior esquerdo em universitários de 18 a 25 anos, segundo o sexo, São Paulo, 1988

\begin{tabular}{lcccccc}
\hline Dentes & \multicolumn{2}{c}{ Ma scul i no } & \multicolumn{2}{c}{ Fe mini no } & \multicolumn{2}{c}{ A mbos } \\
& $\begin{array}{c}\text { Todos os } \\
\text { dentes }\end{array}$ & $\begin{array}{c}\text { Dois } \\
\text { Hemiar }\end{array}$ & $\begin{array}{c}\text { Todos } 0 \text { s } \\
\text { dentes } \\
\%\end{array}$ & $\begin{array}{c}\text { Dois } \\
\text { Hemiar }\end{array}$ & $\begin{array}{c}\text { Todos os } \\
\text { dentes } \\
\%\end{array}$ & $\begin{array}{c}\text { Hemiar } \\
\%\end{array}$ \\
\hline Cariados & 13,6 & 13,7 & 7,3 & 5,6 & 9,6 & 8,9 \\
Obturados & 85,7 & 85,2 & 91,9 & 93,2 & 89,6 & 90,0 \\
Extraídos & 0,5 & 0,8 & 0,8 & 1,2 & 0,8 & 1,0 \\
Ext. Ind. & 0,2 & 0,3 & 0,0 & 0,0 & 0,0 & 0,1 \\
\hline CPO & 100,0 & 100,0 & 100,0 & 100,0 & 100,0 & 100,0 \\
\hline
\end{tabular}

Pelos dados da Tabela 2 podemos verificar que as médias do índice CPO observadas nos hemiarcos superior direito e inferior esquerdo são muito proximas aos resultados dos outros dois hemiarcos (superior esquerdo e inferior direito), evidenciando ter a cárie uma característica bilateral, também na faixa etária dos 18 aos 25 anos. Estes resultados permitem estimar o índice CPO pelo dobro do valor dos dados dos hemiarcos superior direito e inferior esquerdo. As diferenças entre as médias, para cada sexo e para ambos os sexos, são da ordem de um décimo, valores muito baixos, que permitem aceitar simplificação do índice CPO pelo exame de apenas dois hemiarcos. Esta observação concorda com estudo anterior ${ }^{3}$, nas idades de 7 a 12 anos e permite uma economia de tempo e esforço, da ordem de $40 \%$, em levantamentos epidemiológicos de cárie dentária, tornando o custo mais acessível, praticamente sem perda na qualidade e significado da informação obtida.

Para o planejamento e avaliação de programas odontológicos, são necessários dados da composição percentual, apresentados na Tabela 3.

As maiores diferenças entre valores da composição percentual do índice $\mathrm{CPO}$ foram observadas nos dentes cariados, seguido dos obturados, no sexo feminino; as diferenças são menores no sexo masculino, em ambos os sexos, se compararmos dados do feminino.

\section{TABELA 4}

Indice CPO e em seis dentes em universitários de 18 a 25 anos, segundo o sexo. São Paulo, 1988

\begin{tabular}{|c|c|c|c|c|}
\hline \multirow[b]{2}{*}{ Sexo } & \multicolumn{2}{|c|}{ Indice CPO Observ. } & \multirow[b]{2}{*}{$\begin{array}{c}\text { CPO } \\
\text { estudado* }\end{array}$} & \multirow{2}{*}{$\begin{array}{l}\text { Dif CPO } \\
\text { Observ. } \\
\text { estudado }\end{array}$} \\
\hline & $\begin{array}{l}\text { Todos os } \\
\text { dentes }\end{array}$ & $\begin{array}{c}\text { Seis } \\
\text { dentes }\end{array}$ & & \\
\hline Masc. & 13,3 & 3,2 & 12,8 & 0,5 \\
\hline Fem. & 13,7 & 3,5 & 14,0 & $-0,3$ \\
\hline Ambos & 13,5 & 3,4 & 13,6 & $-0,1$ \\
\hline
\end{tabular}

* Média em seis dentes $x 4$

$\mathrm{Na}$ Tabela 4 apresentamos os dados obtidos em seis dentes, e o índice CPO estimado pelo quádruplo da média observada.

Pelos resultados da Tabela 4 observamos que a maior diferença entre o índice CPO e o valor estimado pelos seis dentes ocorreu no sexo masculino, sendo igual a 0,5 dentes; as diferenças, embora maiores do que as propiciadas pelos dois hemiarcos, ainda são baixas e permitem estimar o índice CPO.

Calculamos o coeficiente de correlação entre o índice CPO e os índices observados em dois hemiarcos e seis dentes, na forma descrita por Vieira ${ }^{16}$. Os coeficientes foram respectivamente iguais a 


\section{TABELA 5}

Coeficiente de Prevalência de Cárie (CPC) em universitários de 18 a 25 anos segundo o sexo e dentes examinados, São Paulo, 1988

\begin{tabular}{lccccc}
\hline \multirow{2}{*}{ Sexo } & \multicolumn{2}{c}{ CPC } & \multicolumn{2}{c}{ Diferenças CPC* } \\
\cline { 2 - 6 } & Todos os dentes & Dois Hemiarcos & Dois dentes & Dois Hemiarcos & Seis dentes \\
\hline Masculino & 45,2 & 44,2 & 53,7 & 1,0 & $-8,5$ \\
Feminino & 48,2 & 48,7 & 60,3 & $-0,5$ & $-12,1$ \\
Ambos & 47,1 & 46,8 & 57,6 & 0,3 & $-10,5$ \\
\hline
\end{tabular}

* Em relação a todos os dentes

0,97 (dois hemiarcos) e 0,87 (seis dentes), valores que indicam correlação positiva elevada, próxima da correlação perfeita positiva, que é igual $a+1,0$.

Os valores apurados para a variância das variáveis foram respectivamente iguais a 23,33 (todos os dentes), 9,03 (dois hemiarcos) e 1,84 (seis dentes), evidenciando uma tendência decrescente em função do menor número de dentes examinados em cada unidade amostral. A comparação entre as variâncias deve considerar o quadrado das constantes utilizadas para estimar o índice CPO (2 para dois hemiarcos e $4^{2}$ para seis dentes), 0 que eleva os valores da variância para 36,12 (dois hemiarcos) e 29,44 (seis dentes), valores mais elevados que 23,33 observado para todos os dentes.

O Coeficiente de Prevalência de Cárie (CPC) foi estimado pelos dois hemiarcos e seis dentes. $\mathrm{Na}$ Tabela 5 estảo apresentados os dados desses coeficientes segundo o sexo.

A comparação dos dados da Tabela 5 permite observar que as estimativas do CPC, calculadas pelo exame de dois hemiarcos, apresentam diferenças menores do que as obtidas em seis dentes que determinaram distorções maiores em relação ao coeficiente obtido em todos os dentes.

No presente trabalho os dados dos dois hemiarcos e seis dentes foram obtidos a partir da ficha clínica de cada pessoa examinada, que contém dados para cada um dos dentes, não sendo realizados exames clínicos separados para estas variáveis, o que foi feito por vários autores citados na bibliografia. Talvez possam ocorrer variações pelo exame clínico específico de dois hemiarcos ou seis dentes; não dispomos de elementos para verificar este aspecto, o que deverá ser estudado oportunamente. Devemos considerar, no entanto, que os resultados obtidos e as pequenas diferenças entre 0 índice CPO e o valor estimado para o índice, bem como a composição percentual, são importantes e preliminares para a realização de outro estudo que poderá incluir aspectos específicos sobre exames independentes, calibração de examinadores e estudos de tempo e custo. Por outro lado, se as diferenças fossem grandes, o interesse pela simplificaçăo seria invalidado já no presente estudo.

\section{CONCLUSÖES}

Analisando os dados apresentados apenas por métodos da estatística descritiva, parece ser evidente que a simplificação do índice CPO, baseada no exame de dois hemiarcos oferece estimativas proximas dos valores observados do índice CPO, em relação a média, na faixa etária dos 18 aos 25 anos; o mesmo ocorre, com a composição percentual e o CPC.

Quando se examina apenas os seis dentes selecionados, as estimativas da média são muito razoáveis, embora apresentem diferenças maiores que as obtidas em dois hemiarcos. Em relação ao CPC estimado pelos seis dentes, as distorçőes são maiores, chegando a $12,1 \%$. 
GUIMARÄES, L. O. C. \& GUIMARÃES, A. M. R. [DMF Index simplification from 18 to 25 years]. Rev. Saúde públ., S.Paulo, 24: 407-11, 1990.

ABSTRACT: The DMF index can be estimated at double the value observed in the exam of two hemiarchs (Upper Right and Lower Left), or at four times the value obtained from six teeth (First Molar and Central Incisor Upper Right, first bicuspid upper left, second molar and lateral incisor lower left and second bicuspid lower right. The estimates were made by the use of methods of descriptive statistics, averages, percentages and the coefficient of the prevalence of caries, in a sample of 100 people, aged from 18 to 25 years.

KEYWORDS: DMF index. Dental caries, epidemiology.

\section{REFERÊNCIAS BIBLIOGRÁFICAS}

1. CHAVES, M. M. Odontologia social. $3^{\mathrm{a}}$ ed. Rio de Janeiro, Labor, 1977. p. 29-93.

2. EAST, B. R. \& POHLEN, K. Health index of the teeth. J. dent. Res., 20: 533-41, 1941.

3. GUIMARÃES, L. O. C. Contribuição para o estudo de métodos para estimar o índice $\mathrm{CPO}$, em crianças de 7 a 12 anos. São Paulo, 1969. [Tese de Doutoramento - Faculdade de Odontologia da USP].

4. GUTMARÃES, L. O. C.; MOREIRA, B. H. W.; VIEIRA, S. Estudo da distribuição do coeficiente de prevalência de cárie dentária (CPC). Bol. Ofic. sanit. pana. mer., 53: 24-30, 1974.

5. HEER, A. Apud CHAVES, M. M. ${ }^{1}$

6. KLEN, H. \& PALMER, C. E. Dental caries in American indian children. Publ. Hlth Bull., 239:1-54, Dec. 1937.

7. KNUTSON, J. W. \& KLEIN, H. Studies on dental caries. IV - Tooth mortality in elementary school children. Publ. Hlth Rep., 53: 1021-32, 1938.

8. KNUTSON, J. W. An index of the prevalence of dental caries in school children. Publ. Hlth Rep., 59:253$63,1944$.

9. KNUTSON, J.W. \& ARMSTRONG, W.D. The effect of topically applied sodium fluoride on dental caries experience. II - Report of finding for the second year study. Publ. Hlth Rep., 60: 1085-90, 1945.

10. LILIENTHAL, B. \& ANDREWS, N. A. Applicability of Knutson's index of prevalence of dental caries to data for Australian, Formosan and Indonesian children. Arch. oral Biol., 3: 207-16, 1961.

11. LOPES, E. S. The normality of the distribution in the sample when Viegas simplified index for dental caries ex- perience is used. J.publ.Hlth Dent., 34: 171-81, 1974.

12. PANCOVA, V. \& MRKLAS, H. Caries susceptibility of different types of permanent teeth. Cesk. stomat., 61: $430-62,1961$.

13. RODRIGUES, C. R. M. D. Simplificação do índice de cárie, do índice gengival e do índice de higiene bucal, na faixa etária de 4 a 10 anos (dentições decídua e mista): proposição para o seu uso. São Paulo, 1987. [Dissertação de Mestrado — Faculdade de Odontologia da USP]

14. SCOTT, D. B. Study of bilateral incidence of carious lesions. J. dent. Res., 23: 105-12, 1945.

15. VIEGAS, A. R. Indice simplificado para estimular a prevalência de cárie dental em crianças de 7 a 12 anos de idade. São Paulo, 1968. [Tese de Cátedra — Faculdade de Higiene e Saúde Pública da USP].

16. VIEIRA, S. Introduçäo d̀ bioestatística. Rio de Janeiro, Ed. Campos, 1981. p. 77-89.

17. WELANDER, E. Partial recording of dental caries. Acta odont. scand., 18: $377-85,1960$.

18. WORLD HEALTH ORGANIZATION. Oral health sur. veys: basic methods. Geneva, 1971.

19. WORLD HEALTH ORGANIZATION. Oral health surveys: basic methods. $2^{\text {nd }}$ ed. Geneva, 1977.

20. WORLD HEALTH ORGANIZATION. Oral health surveys: basic methods. $3^{\text {rd }}$ ed. Geneva, 1987.

Recebido para publicação em 10/11/1989

Reapresentado 7/5/1990

Aprovado para publicação em 28/5/1990 